\title{
Acoustic Eigenanalysis with Radial Basis Functions
}

\author{
L. MAJKuT* AND R. Olszewski \\ AGH University of Science and Technology, Department of Mechanics and Vibroacoustics \\ Al. A. Mickiewicza 30, 30-059 Krakow, Poland
}

\begin{abstract}
The paper concerns the eigenanalysis of acoustic cavities with the use of radial basis functions (RBF). The Kansa collocation method was used for determination of the natural frequencies and eigenvectors of $1 \mathrm{D}, 2 \mathrm{D}$ and $3 \mathrm{D}$ acoustic fields. Due to validation analysis of the proposed method, in simple examples like 1D, 2D rectangle and 3D rectangular parallelepiped all calculated eigenferquency and eigenvectors were compared with exact (analytical) results. All results indicate that using of multiquadric radial basis functions provide a results with very high accuracy in comparison to analytical results. In the paper a new method for determining the shape parameter of the multiquadric radial basis functions is described.
\end{abstract}

DOI: 10.12693/APhysPolA.125.A-77

PACS: $02.30 . \mathrm{Mv}, 43.10 . \mathrm{Ks}$

\section{Introduction}

A acoustic characterization of complex cavities is of primary importance in many engineering applications. For example, in room acoustics a prediction of sound level can help in optimization of the acoustic adaptation process, while in the automotive industry, there is a strong demand for methods which can predict the noise level inside a vehicle. In fact, the acoustic eigenvalues analysis is now considered as a necessary step in the early stage of design work on concert halls, class rooms and even vehicles.

Mathematical modeling of acoustic fields in the interior problem results in set of partial differential equations (PDEs) along with a set of boundary (and initial) conditions. In order to deal with complex geometry of acoustic cavities involved in these problems, numerical methods were developed over the last three decades, since exact solutions are usually not available. Traditional numerical approaches for approximate solution of these problems require the use of a mesh: a domain mesh in case of domain methods such as the finite difference method (FDM), the finite element method (FEM), or finite volume method (FVM), or a boundary mesh for boundary methods such as the boundary element method (BEM).

The extraordinary amount of work, which has been put into FEM research since its early days made FEM the dominant approach for most problems in computational mechanics including acoustics. The main difficulties associated with the use of a mesh consist in defining the mesh itself. The generation of a finite element grid with several thousand nodes and element of various sizes, shape and orientation in not a trivial task. Usually the researcher spends the majority of his or her time in creating the mesh, and it becomes a major component of the cost of a simulation process because the cost of CPU time is drastically decreasing.

*corresponding author; e-mail: majkut@agh.edu.pl
Meshless methods, i.e. the methods without employing the concept of element, have been suggested for problems in computational mechanics, as they do not require a mesh to discretize the domain of interest and the approximate solution is constructed entirely in terms of scattered nodes.

Meshless methods may be categorized into two groups: domain type methods such as element-free Galerkin method [1], reproducing Kernel particle [2], the point interpolation method [3-5]; and boundary type methods such as the boundary node method [6], and the boundary point interpolation method $[7,8]$. In these two types of meshless methods the problem domain or only boundary of the domain is discretized by scattered points. In particular, the above-mentioned methods are "meshless" only in terms of interpolation of the domain or boundary variables, as compared to the usual FEM or BEM. Most of the meshless methods have to use the background cells to integrate a weak form over the problem domain. The requirement of the background cells makes a method not "truly" meshless.

Recently, the development in applying the radial basis functions (RBFs) as a truly meshless method of finding approximate solutions of differential equations has drawn the attention of many researchers in science and engineering. What is more, their implementations is more simpler compared to the mainstream numerical techniques such as FDE, FEM, FVM and BEM.

In 1990 Kansa [9] introduced the RBFs collocation method for solving elliptical, hyperbolic and parabolic PDEs. Over the years, this method was extended to solve various ordinary and PDEs including the biphasic and triphasic mixture models for tissue engineering problems [10, 11], 1D nonlinear Burgers' equation [12], shallow water equation for tide and current simulation [13], heat transfer problems [14], free boundary problems [15], Navier-Stokes equations [16], different kinds of Dirichlet [17] and Poisson [18] problems, electromagnetic problems [19], PDE-constrained optimization problems [20] and many others. RBF method succeed in very general settings by composing a univariate function with the Eu- 
clidean norm which turns a multidimensional problem into one-dimensional one.

This paper concerns application of the multiquadric (MQ) RBS to eigenanalysis of 1D, 2D and 3D acoustic fields. For the purpose of validation of the proposed method, all eigenferquency and eigenvectors calculated for simple examples like $1 \mathrm{D}, 2 \mathrm{D}$ rectangle and $3 \mathrm{D}$ rectangular parallelepiped are compared with exact (analytical) results. In the paper a new methods for determining the shape parameter is described.

The paper is organized as follows: in Secs. 2 and 3, a brief introduction to RBF itself and their use in Kansa method is given. For sake of simplicity, the authors begin with describing a $1 \mathrm{D}$ case in Sec. 4 . In Sec. 5 application of the proposed method to a $2 \mathrm{D}$ problem is presented. In Sec. 6, a 3D steady-state acoustic field is considered. Finally, in Sec. 7 of the paper summary and conclusions are given.

\section{RBF}

The Radial Function is a continuous univariate function that has been realized by composition with the Euclidean norm on $\mathbb{R}^{d}$ and can be written as

$$
\phi_{j}(r)=\phi\left(\left\|x-x_{j}\right\|\right),
$$

where $r=\left\|x-x_{j}\right\|$ is the Euclidean distance between points $x$ and $x_{j}$. This radial function can be translated (shifted) and placed on a set of distinct and scattered points $x_{j}$ as its centers to form a family of independent functions.

In this way one single basic RBF, Eq. (1), generates the basis of any function approximation.

Different types of RBFs functions $\phi(r)$ may be categorized into three main groups: compactly supported and finitely smooth; global and finitely smooth; global, infinitely differentiable, containing a free parameter called the shape parameter (denoted by $c$ ).

TABLE I

Definition and class of smoothness of compactly supported Wenland's RBFs.

\begin{tabular}{c|l|c}
\hline \hline Dim. & Definition & Class \\
\hline \hline $\mathrm{d}=1$ & $\phi(r)=(1-r)_{+}$ & $C^{0}$ \\
\cline { 2 - 3 } & $\phi(r)=(1-r)_{+}^{3}(3 r+1)$ & $C^{2}$ \\
\cline { 2 - 3 } & $\phi(r)=(1-r)_{+}^{5}\left(8 r^{2}+5 r+1\right)$ & $C^{4}$ \\
\hline \multirow{5}{*}{$\mathrm{d}=2,3$} & $\phi(r)=(1-r)_{+}^{2}$ & $C^{0}$ \\
\cline { 2 - 3 } & $\phi(r)=(1-r)_{+}^{4}(4 r+1)$ & $C^{2}$ \\
\cline { 2 - 3 } & $\phi(r)=(1-r)_{+}^{6}\left(35 r^{2}+18 r+3\right)$ & $C^{4}$ \\
\cline { 2 - 3 } & $\phi(r)=(1-r)_{+}^{8}\left(32 r^{3}+25 r^{2}+8 r+1\right)$ & $C^{6}$
\end{tabular}

Table I list some of compactly supported CS-RBFs constructed by Wendland [21]. It contains the lowest possible degree among all piecewise polynomial CSRBFs which are positive defined on $\mathbb{R}^{d}$ for given order of smoothness, where

$$
(1-r)_{+}^{n}= \begin{cases}(1-r)^{n}, & \text { if } r \in\langle 0,1\rangle \\ 0 & \text { if } r>1\end{cases}
$$

Globally supported RBFs.

TABLE II

\begin{tabular}{l|l}
\hline \hline Definition & Name of RBF \\
\hline \hline$\phi(r)=r$ & linear \\
\hline$\phi(r)=r^{3}$ & cubic \\
\hline$\phi(r)=\sqrt{r^{2}+c^{2}}$ & MQ \\
\hline$\phi(r)=r^{2} n \log r$ & thin plate spline (TSP) \\
\hline$\phi(r)=\frac{1}{\sqrt{r^{2}+c^{2}}}$ & inverse MQ \\
\hline$\phi(r)=\exp ^{-c r^{2}}$ & Gaussian (GA)
\end{tabular}

Other compactly supported RBF have been proposed by $\mathrm{Wu}$ [22] and Gneiting [23].

Table II lists some commonly used, globally supported RBFs.

In this paper we focus on the MQ RBF due its popularity in applications and its good approximation properties. The MQ function with center at $x_{j}$ may be written in the form:

$$
\begin{aligned}
& \phi_{j}(r)=\sqrt{\left(x-x_{j}\right)^{2}+c_{j}^{2}} \\
& \quad \text { in } 1 \text { D problems } \\
& \phi_{j}(r)=\sqrt{\left(x-x_{j}\right)^{2}+\left(y-y_{j}\right)^{2}+c_{j}^{2}} \\
& \quad \text { in } 2 \mathrm{D} \text { problems } \\
& \phi_{j}(r)=\sqrt{\left(x-x_{j}\right)^{2}+\left(y-y_{j}\right)^{2}+\left(z-z_{j}\right)^{2}+c_{j}^{2}}
\end{aligned}
$$$$
\text { in } 3 \mathrm{D} \text { problems. }
$$

The MQ (2) is a representative of the group of RBFs that are global, infinitely differentiable and contain the shape parameter $c$.

In MQ RBF meshless method, the shape of the basis function (2) is controlled by a free parameter $c$. As $c$ gets larger, the shape becomes flat and is insensitive to the difference in Euclidean (radial) distance. The choice of the shape parameter $c$ is a difficult and so far unresolved problem.

\section{Kansa's method for solving differential equations}

The Kansa's collocation method is one of the meshless (meshfree) methods, which are based on approximation and collocation of global functions. Unlike the FEM, which is based on patching together elements with local, low-degree polynomial interpolants, the global shape function extend their influence to the entire domain of interest, without division into elements.

Consider the following governing equation:

$$
L u=f(x) \quad x \in \Omega,
$$

subject to the boundary condition

$$
B u=g(x) \quad x \in \Gamma,
$$

where $L$ is a linear differential operator, $B$ is a boundary operator of order lower than $L, \Omega$ is the analyzed domain and $\Gamma$ is the boundary of the domain.

The general idea of Kansa's method is to approximate the solution of the problem (3) and (4) in such a way that it can be represented as a sum of a series of globally supported RBFs: 


$$
\widehat{u}=\sum_{j=1}^{N} \alpha_{j} \phi_{j}(r),
$$

where $\phi_{j}(r)$ are globally supported RBFs (in this paper only MQ RBFs (2) are used) and $\alpha_{j}$ are constant coefficients to be determined by collocation procedure.

The approximate solution (5) must satisfy the governing equation (3) and boundary condition (4). Lets us assume that on a set of $N_{I}$ distinct (collocation) points $\left\{x_{1}, x_{2}, \ldots, x_{N_{I}}\right\} \in \Omega$, the approximate solution is required to satisfy the the governing equation (3):

$$
\begin{gathered}
\widehat{u}=f(x) \Leftrightarrow \sum_{j=1}^{N} \alpha_{j} L \phi_{j}\left(r_{i}\right)=f\left(x_{i}\right) \\
i=1,2, \ldots, N_{I} .
\end{gathered}
$$

By the same token, for a set of $N_{B}$ points on the boundary $\left\{x_{N_{I}+1}, x_{N_{I}+2}, \ldots x_{N_{I}+N_{B}} \in \Gamma\right.$ it is required that

$$
\begin{aligned}
& \sum_{j=1}^{N} \alpha_{j} B \phi_{j}\left(r_{i}\right)=g\left(x_{i}\right) \\
& \quad i=N_{I}+1, N_{I}+2, \ldots, N_{I}+N_{B} .
\end{aligned}
$$

Eqs. (6) and (7) constitute linear systems, which can be written in matrix form

$$
A \alpha=f .
$$

The system (8) can be solved for coefficients $\alpha_{j}, j=$ $1,2, \ldots, N$. In case when $N_{I}+N_{B}=N$, the Gaussian elimination or inverse matrix procedure can be used, when $N_{I}+N_{B}>N$, the overdetermined linear system can be solved in the last square sense.

The choice of the shape parameter $c$ is the source of conflict between theoretically achievable accuracy and numerical stability. The error in interpolation decrease when the shape parameter increase but then the condition number of matrix $\boldsymbol{A}$ increase, i.e. the collocation matrix becomes ill-conditioned. In literature this phenomenon has been referred to as the trade-off or uncertainty principle [24].

The extend review of choosing of the "optimal" shape parameter in given in [25]. In this paper a new method of determining of the shape parameter is proposed.

Once the coefficients $\alpha_{j}$ are determined the approximate solution is given by (5), which is defined for all $x \in \Omega$. So reconstructed solution in with MQ RBFs, at evaluation point $x_{e}$ can be written as

$$
\begin{aligned}
& \widehat{u\left(x_{e}\right)}=\sum_{j=1}^{N} \alpha_{j} \sqrt{\left(x_{e}-x_{j}\right)^{2}+c^{2}} \\
& \quad \text { in } 1 \mathrm{D} \text { problems } \\
& \widehat{u\left(x_{e}\right)}=\sum_{j=1}^{N} \alpha_{j} \sqrt{\left(x_{e}-x_{j}\right)^{2}+\left(y_{e}-y_{j}\right)^{2}+c^{2}} \\
& \quad \text { in } 2 \mathrm{D} \text { problems } \\
& \widehat{u\left(x_{e}\right)}=\sum_{j=1}^{N} \alpha_{j} \\
& \quad \times \sqrt{\left(x_{e}-x_{j}\right)^{2}+\left(y_{e}-y_{j}\right)^{2}+\left(z_{e}-z_{j}\right)^{2}+c^{2}} \\
& \quad \text { in 3D problems. }
\end{aligned}
$$

In all cases analyzed here, constant shape parameter will be assumed i.e. $c_{j}=c$ - one value for all basis functions.

\section{1. $M Q R B F$ solution of the acoustic field}

Considering an acoustic cavity (domain $\Omega$ ) filed with a perfect fluid and implicit $e^{i \omega t}$ time dependance, the harmonic wave propagation problem in terms of acoustic pressure amplitude in governed by the Helmholtz equation in form:

$$
\nabla^{2} p(r)+k^{2} p(r)=0 \quad r \in \Omega \subset \mathbb{R}^{d},
$$

where $\nabla$ is the Nabla operator, $p$ is the amplitude of the acoustic pressure, $k$ is the wave number $(\omega / s), \omega$ is the circular frequency, $s$ is the sound velocity in fluid medium and $d=1,2$ or 3 is domain dimension. The acoustic problem consist in computing eigenvalues (wave number $k$ ) in the fluid within the enclosure with prescribed boundary conditions.

According to Kansa'a method, Eq. (10) can be written as

$$
\left.\sum_{j=1}^{N} \alpha_{j} \nabla^{2} \sqrt{r_{j}^{2}+c^{2}}\right|_{r=r_{i}}+k^{2} \sum_{j=1}^{N} \alpha_{j} \sqrt{r_{j}^{2}+c^{2}}=0,
$$

where $r_{i}$ are coordinates of collocation points $i=$ $1,2, \ldots, N_{I}$, and $r_{j}$ are coordinates of RBF centers $j=$ $1,2, \ldots, N$

$$
\begin{aligned}
r_{j}^{2}= & \left(x_{i}-x_{j}\right)^{2}, \\
& \text { in } 1 \mathrm{D} \text { problems } \\
r_{j}^{2}= & \left(x_{i}-x_{j}\right)^{2}+\left(y_{i}-y_{j}\right)^{2}, \\
& \text { in } 2 \mathrm{D} \text { problems } \\
r_{j}^{2}= & \left(x_{i}-x_{j}\right)^{2}+\left(y_{i}-y_{j}\right)^{2}+\left(z_{i}-z_{j}\right)^{2}, \\
& \text { in } 3 \mathrm{D} \text { problems. }
\end{aligned}
$$

For each $i$ Eq. (11) gives $i-t h$ row of collocation matrix $\boldsymbol{A}$ (8) (now the vector $\boldsymbol{f} \equiv 0$ ). The last $N_{B}$ rows of this matrix depends on boundary conditions (set of $N_{B}$ points on the boundary).

To obtain the nontrivial solution $(\alpha \neq 0)$, the determinant of the matrix $\boldsymbol{A}$ must be zero, i.e. $\operatorname{det} \boldsymbol{A}=0$. From this equation, the natural frequencies of the beam can be calculated.

\subsection{Choice of the shape parameter}

The simplest strategy is perform a series of approximation experiments with varying shape parameter and then pick the "best" one. It is possible only in case when the solution of the differential equation in question is known. Other popular strategies are based on the power function, cross validation, or Contour-Pade algorithm. All of them are described in [24].

Herein, the authors propose to introduce another strategy, which is based on following algorithm:

1. Establishing a range of shape parameter $c$ with step $\Delta c$,

2. Finding $k$ values for which $\operatorname{det} \boldsymbol{A}=0$,

3. If there is no indication of stable value of $k$ for various $c$, then going to 1 (and changing the range of $c$ ); 
4. If there is a stable (the same) value of $k$ for various $c$, choosing any value of $c$ from this stable range,

In Fig. 1a, the variable $k$ as a function of shape parameter $c$ is shown, whereas the rage of shape parameter was chosen as $c \in\langle 0.01-1.2\rangle$ with step $\Delta c=0.01$. It is clearly visible from this figure that, for wide range of $c$ from about 0.2 to about 0.9 , there is only one (stable) value of $k$ obtained from calculation.

Any value from this rage can be chosen as "optimal" in RBF analysis. There is no problem with condition number of collocation matrix, because there is clearly visible in Fig. 1 when (for which value of $c$ ) the solution breaks down.

In Fig. 1b solution of equation $\operatorname{det} \boldsymbol{A}=0$ for a wider rage of $k$ is shown. Six successive wave numbers are visible In this figure.
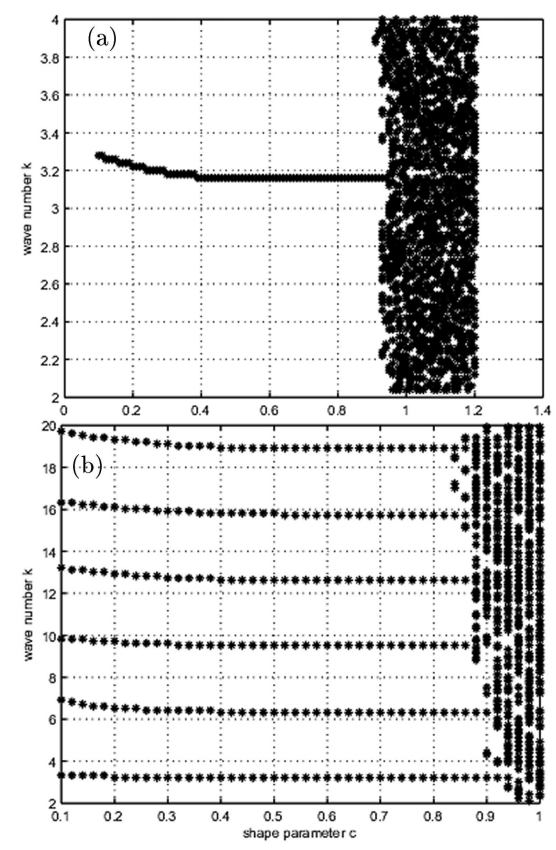

Fig. 1. An example of using the proposed algorithm of choosing the "optimal" shape parameter: (a) only one (first) eigenvalue. (b) six successive eigenvalues.

\section{One-dimensional acoustical field}

Using the collocation method for solving the Helmholtz equation (10) leads to system of equation in the form:

$A \alpha=0$.

Here the elements of matrix $\boldsymbol{A}$ are given by

$$
\begin{aligned}
& A_{i, j}=\left.\frac{\mathrm{d}^{2}}{\mathrm{~d} x^{2}}\right|_{x=x_{i}} \sqrt{\left(x-x_{j}\right)^{2}+c^{2}} \\
& +k^{2} \sqrt{\left(x_{i}-x_{j}\right)^{2}+c^{2}} .
\end{aligned}
$$

The last two rows of matrix $\boldsymbol{A}$ depend on boundary conditions

$$
A_{i, j}=\left.\frac{\mathrm{d}}{\mathrm{d} x}\right|_{x=0, l} \sqrt{\left(x-x_{j}\right)^{2}+c^{2}} .
$$

Components of the vector $\alpha=\left[\alpha_{1}, \alpha_{2}, \ldots, \alpha_{N}\right]^{T}$ represent the "magnitudes" of the $j$-th RBF function.

To obtain a nontrivial solution $(\alpha \neq 0)$, the determinant of the matrix $\boldsymbol{A}$ must be zero, i.e. $\operatorname{det} \boldsymbol{A}=0$. From this equation, the eigenvalues $k$ can be calculated.

\subsection{Comparison of results}

For the purpose of validation of the proposed method, in a simple 1D example all calculated eigenfrequency and eigenvectors are compared with exact (analytical) results. The following relative errors were used to measure the quality of the numerical solution (superscript 'exact' denotes analytical results, superscript 'RBF' - approximation with $\mathrm{RBF}$ ).

$$
E_{\omega_{i}}=\sqrt{\frac{\left(\omega_{i}^{\text {exact }}-\omega_{i}^{R B F}\right)^{2}}{\left(\omega_{i}^{\text {exact }}\right)^{2}}} \cdot 100 \%,
$$

where $\omega_{i}$ is the $i$-th natural frequency of the $1 \mathrm{D}$ acoustic field, and

$$
E_{i}^{\text {mode }}=\sqrt{\frac{\int_{0}^{l}\left(X_{i}^{\text {exact }}-X_{i}^{R B F}\right)^{2} \mathrm{~d} x}{\int_{0}^{l}\left(X_{i}^{\text {exact }}\right)^{2} \mathrm{~d} x}} \cdot 100 \%,
$$

where the $X_{i}$ is the normalized $i$-th mode shape of the cavity. In all calculations presented in this section $N=200$ (the number of evaluation points along the analyzed cavity) is used.

The values of errors $E_{\omega_{i}}$ and $E_{i}^{\text {mode }}$ computed for the first 25 eigenvalues $(i=1,2, \ldots 25)$ are shown in Fig. $2 \mathrm{a}$ and Fig. $2 \mathrm{~b}$ respectively.

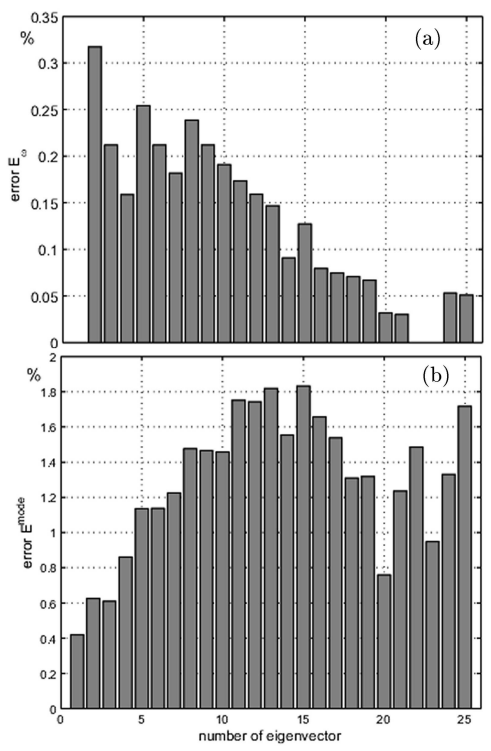

Fig. 2. Errors in MQ RBF eigenanalysis of $1 D$ acoustic field: (a) errors in eigenfrequencies calculation (b) errors in eigenvectors determination. 
The values of errors shown in Fig. 2 suggest that the results of the $1 \mathrm{D}$ acoustic field with MQ RBF are more than very similar to analytical results.

\section{Two-dimensional acoustic field}

In case of $2 \mathrm{D}$ acoustic field, the first $N_{I} \times N$ elements ( $N_{I}$ is a number of collocation points, $N$ is a number of MQ RBF functions used in approximation) of the approximation matrix $\boldsymbol{A}$ (see Eq. (8)) are given by:

$$
\begin{aligned}
& A_{i, j}=\left.\frac{\partial^{2}}{\partial x^{2}}\right|_{(x, y)=\left(x_{i}, y_{i}\right)} \sqrt{\left(x-x_{j}\right)^{2}+\left(y-y_{j}\right)^{2}+c^{2}} \\
& +\left.\frac{\partial^{2}}{\partial y^{2}}\right|_{(x, y)=\left(x_{i}, y_{i}\right)} \sqrt{\left(x-x_{j}\right)^{2}+\left(y-y_{j}\right)^{2}+c^{2}} \\
& +k^{2} \sqrt{\left(x_{i}-x_{j}\right)^{2}+\left(y_{i}-y_{j}\right)^{2}+c^{2}} .
\end{aligned}
$$

The last $N_{B}$ (number of boundary points) rows of matrix $\boldsymbol{A}$ depend on boundary conditions.

$$
A_{i, j}=\left.\frac{\partial}{\partial n}\right|_{x, y \in \Gamma} \sqrt{\left(x-x_{j}\right)^{2}+\left(y-y_{j}\right)^{2}+c^{2}} .
$$

In the above equation $\frac{\partial}{\partial n}$ is the directional derivative of RBF in the direction of the outward pointing normal $n$ to the boundary line $\Gamma$ :

$$
\frac{\partial \phi(r)}{\partial n}=\nabla \varphi(r) \cdot \boldsymbol{n} \text {. }
$$

To obtain a nontrivial solution $(\alpha=0)$, the determinant of the matrix $\boldsymbol{A}$ must be zero i.e. $\operatorname{det} \boldsymbol{A}=0$. From this equation the natural frequencies are calculated.

\subsection{Simple 2D rectangular domain}

For the purpose of validation of the proposed method, firstly a simple $2 \mathrm{D}$ example of acoustic field in a rectangular domain was analyzed. All calculated eigenfrequency and eigenvectors are compared with exact (analytical) results. The following relative errors were used to measure the quality of the numerical solution (superscript 'exact' denotes analytical results and subscript 'RBF' - approximation with RBF.

$$
E_{\omega_{i}}=\sqrt{\frac{\left(\omega_{i}^{\text {exact }}-\omega_{i}^{R B F}\right)^{2}}{\left(\omega_{i}^{\text {exact }}\right)^{2}}} \cdot 100 \%,
$$

where $\omega_{i}$ is the $i$-th natural frequency,

$$
E_{i}^{\text {mode }}=\frac{1}{N_{e}} \sqrt{\frac{\sum_{i=1}^{N_{e}}\left(X_{i}^{\text {exact }}-X_{i}^{R B F}\right)^{2}}{\sum_{i=1}^{N_{e}}\left(X_{i}^{\text {exact }}\right)^{2}}} \cdot 100 \%,
$$

where $X_{i}$ is the normalized $i-t h$ mode shape. In all calculations presented in this section $N_{e}=165$ (the number of evaluation points in the analyzed domain) is used.

The values of errors $E_{\omega_{i}}$ and $E_{i}^{\text {mode }}$ computed for the first 25 eigenvalues $(i=1,2, \ldots 25)$ are shown in Fig. 3a and Fig. 3b respectively.

The values of errors shown in Fig. 3 suggest that the results of the acoustic eigenanalysis in $2 \mathrm{D}$ domain are very similar to analytical ones.

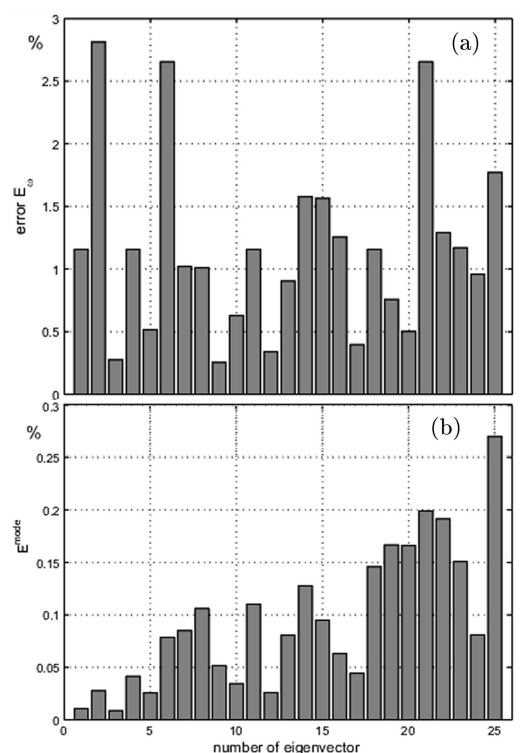

Fig. 3. Errors in MQ RBF eigenanalysis of 2D simple rectangular acoustic field: (a) errors in eigenfrequencies calculation (b) errors in eigenvectors determination.

\subsection{Arbitrary 2D domain}

As an another example, the shape shown in Fig. 4 was adopted as an arbitrary domain.

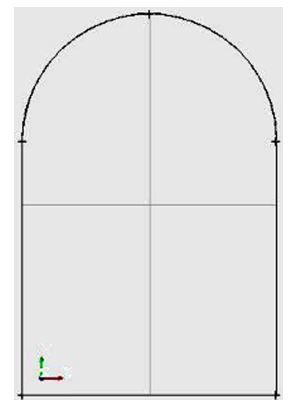

Fig. 4. The analyzed 2D acoustic domain.

The geometrical model was prepared in CAE opensource software SALOME v.5.1.4 [26]. This software was used for geometrical modeling and boundary discretization. The boundary nodes were used by the authors' own software to define coordinates of the MQ RBF centers and collocation points. In Fig. 5 the domain with RBF centers (denoted by "x") and collocation points (denoted by "o") is shown.

All calculated eigenfrequency were compared with finite element analysis results (obtained with the use of SALOME [26] software). The following relative error was used in comparison of results (superscript 'FEM' denotes finite element analysis results and superscript 'RBF' - approximation with $\mathrm{RBF})$ : 


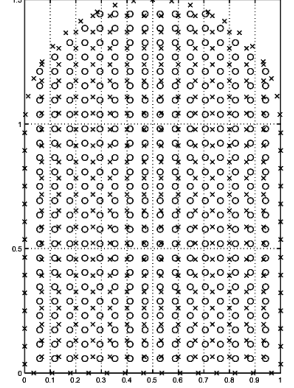

Fig. 5. The analyzed 2D acoustic domain with RBF centers (denoted by $\mathrm{x}$ ) and collocation points (denoted by 0 ).

$$
E_{\omega_{i}}=\sqrt{\frac{\left(\omega_{i}^{F E M}-\omega_{i}^{R B F}\right)^{2}}{\left(\omega_{i}^{F E M}\right)^{2}}} \cdot 100 \%,
$$

where the $\omega_{i}$ is the $i$-th natural frequency.

The values of error $E_{\omega_{i}}$ computed for the first 25 eigenvalues $(i=1,2, \ldots 25)$ are shown in Fig. 6 .

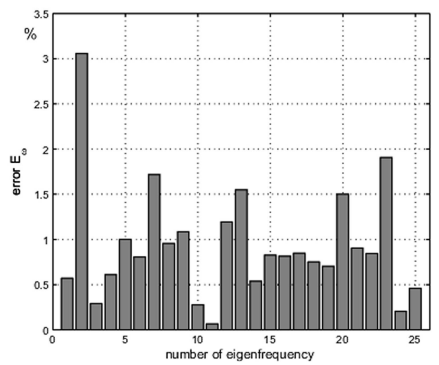

Fig. 6. Errors in MQ RBF eigenfrequencies calculation of $2 \mathrm{D}$ acoustic field.

In Fig. 7, examples of the mode shapes are shown for the 11-th mode shape of which Fig. 7a results of the FEM analysis and Fig. $7 \mathrm{~b}$ - the outcome of RBF analysis.

The values of errors shown in Fig. 6 suggest that the results of the acoustic eigenanalysis in $2 \mathrm{D}$ domain are very accurate (in comparison to FEM analysis), even in case of eigenfrequences near to each other.

\section{Three-dimensional acoustic field}

In case of 3D acoustic field the first $N_{I} \times N$ elements ( $N_{I}$ is a number of collocation points, $N$ is a number of MQ RBF functions used in approximation) of the approximation matrix $\boldsymbol{A}$ (see Eq. (8)) are given by:

$$
\begin{gathered}
A_{i, j}=\left.\frac{\partial^{2}}{\partial x^{2}}\right|_{(x, y, z)=\left(x_{i}, y_{i}, z_{i}\right)} \\
\sqrt{\left(x-x_{j}\right)^{2}+\left(y-y_{j}\right)^{2}+\left(z-z_{j}\right)^{2}+c^{2}} \\
+\left.\frac{\partial^{2}}{\partial y^{2}}\right|_{(x, y, z)=\left(x_{i}, y_{i}, z_{i}\right)}
\end{gathered}
$$

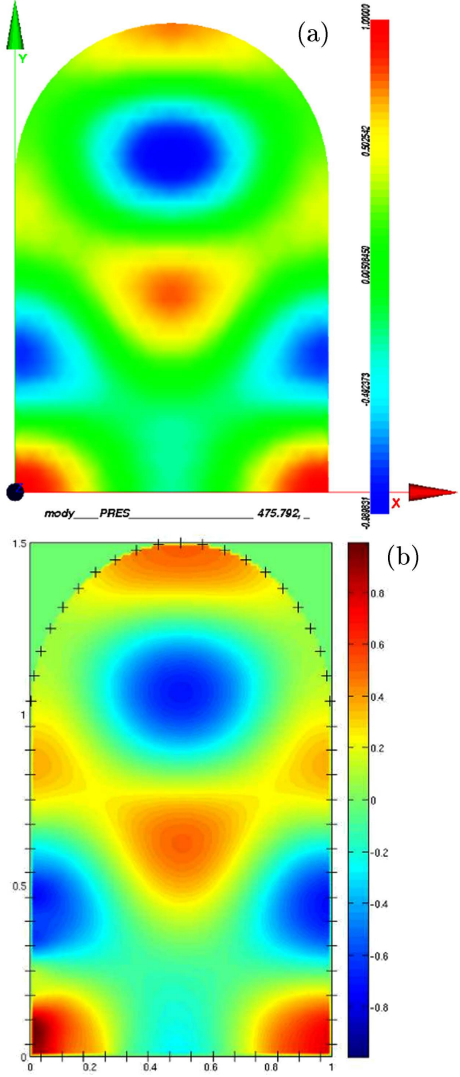

Fig. 7. The 11th mode shape of $2 \mathrm{D}$ acoustic field: (a) result of FEM analysis (b) MQ RBF analysis.

$$
\begin{aligned}
& \sqrt{\left(x-x_{j}\right)^{2}+\left(y-y_{j}\right)^{2}+\left(z-z_{j}\right)^{2}+c^{2}} \\
+ & \left.\frac{\partial^{2}}{\partial z^{2}}\right|_{(x, y, z)=\left(x_{i}, y_{i}, z_{i}\right)} \\
& \sqrt{\left(x-x_{j}\right)^{2}+\left(y-y_{j}\right)^{2}+\left(z-z_{j}\right)^{2}+c^{2}} \\
+ & k^{2} \sqrt{\left(x_{i}-x_{j}\right)^{2}+\left(y_{i}-y_{j}\right)^{2}+\left(z_{i}-z_{j}\right)^{2}+c^{2}} .
\end{aligned}
$$

The last $N_{B}$ (number of boundary points) rows of matrix $\boldsymbol{A}$ depend on boundary conditions:

$$
\begin{aligned}
A_{i, j} & =\left.\frac{\partial}{\partial n}\right|_{x, y, z \in \Gamma} \\
& \sqrt{\left(x-x_{j}\right)^{2}+\left(y-y_{j}\right)^{2}+\left(z-z_{j}\right)^{2}+c^{2}} .
\end{aligned}
$$

In the above equation $\frac{\partial}{\partial n}$ is the directional derivative of RBF in the direction of the outward pointing normal $n$ to the boundary line $\Gamma$ :

$$
\frac{\partial \phi(r)}{\partial n}=\nabla \varphi(r) \cdot \boldsymbol{n}
$$

To obtain a nontrivial solution $(\alpha=0)$, the determinant of the matrix $\boldsymbol{A}$ must be zero i.e. $\operatorname{det} \boldsymbol{A}=0$. From this equation the natural frequencies are calculated. 


\subsection{Comparison of results}

For the purpose of validation of the proposed method, in 3D simple rectangular parallelepiped cavity was analyzed. All calculated eigenfrequency were compared with exact (analytical) results. The following relative error was used to measure the quality of the numerical solution (superscript 'exact' denotes analytical results and superscript 'RBF' - approximation with RBF:

$$
E_{\omega_{i}}=\sqrt{\frac{\left(\omega_{i}^{\text {exact }}-\omega_{i}^{R B F}\right)^{2}}{\left(\omega_{i}^{\text {exact }}\right)^{2}}} \cdot 100 \%,
$$

where $\omega_{i}$ is the $i$-th natural frequency of acoustic field.

The value of error $E_{\omega_{i}}$ computed for the first 25 eigenvalues $(i=1,2, \ldots 25)$ is shown in Fig. 8 .

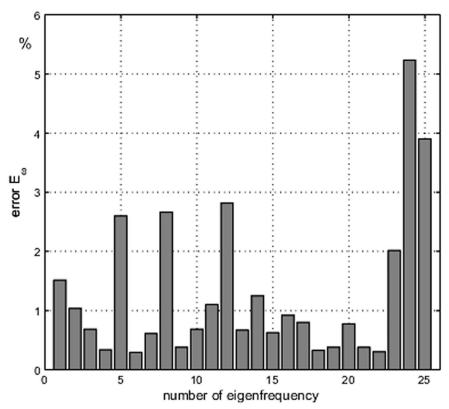

Fig. 8. Errors in MQ RBF eigenanalysis of 3D acoustic field.

The values of errors shown in Fig. 8 suggest that the results of the $3 \mathrm{D}$ acoustic field with MQ RBF are very similar to analytical results.

\section{Summary and concluding remarks}

In this paper, a meshless method for acoustic eigenanalysis in $1 \mathrm{D}, 2 \mathrm{D}$ and $3 \mathrm{D}$ domain is described. The study presented herein concerns applications the MQ RBS to finding approximate solution of the Helmholtz equation, searching for the natural frequency (eigenfrequency) and reconstruction the solution (eigenvectors). In case of $1 \mathrm{D}$ domain, simple $2 \mathrm{D}$ rectangular domain and $3 \mathrm{D}$ rectangular parallelepiped domain, all results have been compared against analytical ones.

The proposed method revealed to be very accuracy in the search of eigenfrequencies even in case of eigenfrequencies near to each other.

\section{References}

[1] T. Belystcho, Y. Lu, L. Gu, Int. J. Numer. Meth. Engng. 37, 229 (1994).

[2] W.K. Liu, S. Jun, Y.F. Zhang, Int. J. Numer. Meth. Engng. 20, 1081 (1995).

[3] Y.T. Gu, G.R. Liu, Comput. Methods Appl. Mech Engrg. 190, 5515 (2001).

[4] G.R. Liu, Y.T. Gu, Int. J. Numer. Methods Engrg 50, 937 (2001).

[5] J.G. Wang, G.R. Liu, Int. J. Numer. Methods Engrg. 54, 1623 (2002).

[6] Y.X. Mukherjee, S. Mukherjee, Int J. Numer. Meth Engng. 40, 797 (1997).

[7] V. Sladek, J. Sladek, S.N. Atluri, R. van Keer, 10.1007/s004660050486 Comput. Mech. 25, 394 (2000).

[8] G.R. Liu, Y.T. Gu, Comput. Mech. 28, 47 (2002).

[9] E.J. Kansa, Comput. Math. Appl. 19, 147 (1990).

[10] Y.C. Hon, M.W. Lu, W.M. Xue, Y.M. Zhu, Appl. Math. Comput. 88, 153 (1997).

[11] Y.C. Hon, M.W. Lu, W.M. Xue, X. Zhou, Comput Mech. 24, 155 (1999).

[12] Y.C. Hon, X.Z. Mao, Appl. Math. Comput. 95, 37 (1998).

[13] Y.C. Hon, K.F. Cheung, X.Z. Mao, E.J. Kansa ASCE J. Hydraul. Engng. 125, 524 (1999).

[14] M. Zerroukat, H. Power, C.S. Chen, Int. J. Numer Meth. Engng. 42, 1263 (1998).

[15] J. Perko, C.S. Chen, B. Sarler, in Moving Boundaries VI, Computational Modelling of Free and Moving Boundary Problems, WIT Press, 2001, p. 111.

[16] P.P. Chinchapatnam, K. Djidjeli, Nair, P.B. Int. J. Comput. Math. 841509 (2007).

[17] Y. Duan, Y. Tan, 0.1016/j.cam.2005.09.018J. Comput. Appl. Math. 196, 394 (2006).

[18] A. Aminataei, M.M. Mazarei, Comput. Math. Appl. 56, 2887 (2008).

[19] P. Vu, G.E. Fasshauer, IET Science, Measurement ETechnology 5, 206 (2011).

[20] J.W. Pearson, Numer. Algor., December (2012).

[21] H. Wendland, Adv. Comput. Math. 4, 389 (1995).

[22] Z. Wu, Adv. Comput. Math. 4, 283 (1995).

[23] T. Gneiting, J. Multivariate Analysis 83, 493 (2002).

[24] G.E. Fasshauer, Meshfree Approximation Methods With MATLAB, World Scientific, Singapore 2007.

[25] G.E. Fasshauer, J.G. Zhang, Numer. Algor.45, 346 (2007).

[26] www.salome-platform.org/. 